\section{A MASK FOR YOUR TOOTHBRUSH}

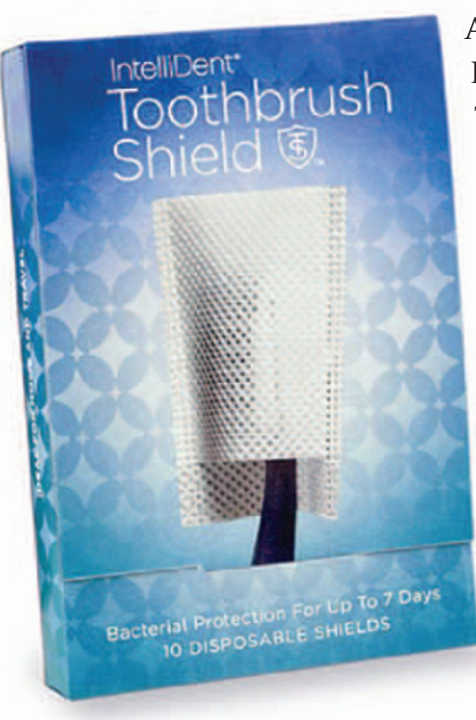

A US company called IntelliDent has launched The Toothbrush

Shield, a disposable, breathable and fastdrying shield that acts like a surgical mask for your toothbrush. It is designed to replace the plastic snap-on caps which have been proven to trap and breed bacteria.

The Toothbrush Shield is made of an antimicrobial non-woven material that wicks away moisture from your toothbrush, creating a cleaner, healthier environment for your toothbrush - and your mouth. It is a laboratory tested 99.9\% effective barrier against bacterial and viral transfer from both airborne and surface microbial contact.

The Toothbrush Shield is available from www. ToothbrushShield.com and IntelliDent plan to launch it in the UK in the near future.

\section{ENTER THE WORLD OF 3D CONE BEAM DIAGNOSTICS}

When practices buy a Galileos Compact from Sident Dental Systems it offers them a convenient entry to the world of 3D cone beam digital diagnostics, with the ability to upgrade for expansion. It also offers unparalleled functionality in diagnosis, planning and treatment because it also includes a CEPH facility.

Galileos 3D cone beam digital $\mathrm{X}$-ray systems offer practices the power of integrated diagnostics and treatment planning via a single imaging system. It enables them to reduce risks, plan surgical interventions, coordinate treatment planning with colleagues and explain treatment to patients with even greater clarity, certainty and ease.

With their innovative cone beam technology, they can calculate a large volume 3D image set (over 200 exposures) in a single low-dose scan lasting 15 seconds or less. This 3D image set is then processed and displayed using their integrated GALAXIS 3D software.

The Galileos Compact presents its 3D images in perfect image quality and facilitates easy navigation and diagnosis in traditional PAN and TSA presentations. The Galileos Comfort offers CEPH views too. Both are all-in-one diagnosis and planning systems which offer a very short, low dose scanning cycle for optimum speed and efficiency.

For further information call 01932582900 , email j.colville@sident.co.uk or visit www.sident.co.uk.

\section{IMAGES UNHINDERED BY TRAILING WIRES}

Schick CDR Elite from Clark Dental produces incredibly high quality images to meet the diagnostic needs of every clinician. To expand even further, Clark Dental now offers Schick WiFi - a new communications platform designed to add portable functionality to the highly versatile Schick system.

Schick WiFi brings a new level of freedom and flexibility to dental digital radiography. The new addition to the Schick family allows for simple room-to-room transfer, and complete $360^{\circ}$ chair access, unhindered by trailing wires and other unnecessary complications.

Schick WiFi is available with all three sizes of CDR Elite sensor, and can also be purchased as an expansion kit for existing Elite users. Furthermore, Schick WiFi is also compatible with several popular imaging software products and practice management systems, meaning existing users and new customers alike can maximise their imaging investment by integrating Schick WiFi on their own terms.

For more information call 01268733146 , emailinfo@clarkdental. co.uk or visit www. clarkdental.co.uk.

\section{EASY TO GET TO} GRIPS WITH

Sirona's CEREC SW 4.0 software's defining features are a sharp focus on essentials, attractive and modern design, and the streamlined production of clinically and aesthetically perfect dental restorations. A key highlight is the intuitive user interface which has been redesigned using the latest software development tools. All the settings and processing steps are visualised with the aid of self-explanatory icons and photorealistic images. As a result, newcomers will find it easy to get to grips with the CEREC system - and experienced users will be able to achieve even more convincing results than in the past.

The CEREC system enables dentists to create allceramic restorations directly 'in-house'. The CEREC SW 4.0 system guides you step by step through the entire production procedure. The software functions and options adapt themselves dynamically as the work proceeds. The user interface remains clear and uncluttered, and only those items that are relevant are displayed at any given time.

To cater for patients with multiple clinical indications the SW 4.0 software allows you to work in parallel on several different restorations during a single treatment session. As a result you are ideally placed to offer your patients a complete portfolio of aesthetic and functionally effective therapy solutions.

Appealing to experienced and new users alike, the software upgrade is available free of charge to CEREC Club members.

For further information telephone 08450715040 or emailinfo@sironadental.co.uk. 